Irish Math. Soc. Bulletin

Number 70, Winter 2012, 35-36

ISSN 0791-5578

\title{
CHARACTERIZING THE SPECTRA OF NONNEGATIVE MATRICES
}

\author{
ANTHONY G. CRONIN
}

This is an abstract of the $\mathrm{PhD}$ thesis Characterizing the Spectra of Nonnegative Matrices written by Anthony Cronin under the supervision of Professor Thomas J Laffey at the School of Mathematical Sciences UCD and submitted in May 2012.

In this thesis we investigate the nonnegative inverse eigenvalue problem (NIEP). This is the problem of characterizing all possible spectra of entrywise nonnegative $n \times n$ matrices. We give a new inequality [1] relating Newton power sums. We then build on perturbation results of Guo [2] and Laffey [3]. Also presented are results for the class of doubly companion matrices and we address some questions of Monov [4]. We examine the classic spectrum $(3+t, 3,-2,-2,-2)$ and give some new results on the diagonalizable and symmetric nonnegative inverse eigenvalue problems (DNIEP and SNIEP respectively). The main results are:

Theorem 1. Let $n>1$ and $A$ be a nonnegative $n \times n$ matrix. Then

$$
\Omega:=n^{2} s_{3}-3 n s_{1} s_{2}+2 s_{1}^{3}+\frac{n-2}{\sqrt{n-1}}\left(n s_{2}-s_{1}^{2}\right)^{\frac{3}{2}} \geq 0,
$$

where $s_{k}=\operatorname{trace}\left(A^{k}\right), k=1,2,3$.

Theorem 2. Suppose that $c$ is a real number such that, for all integers $n \geq 3$, and all lists $\sigma:=\left(\rho, \lambda, \bar{\lambda}, \lambda_{4}, \ldots, \lambda_{n}\right)$ with Perron root $\rho$ and $\lambda \notin \mathbb{R}$, the realizability of $\sigma$ implies the realizability of $\sigma_{c}:=\left(\rho+c t, \lambda+t, \bar{\lambda}+t, \lambda_{4}, \ldots, \lambda_{n}\right)$, then $c \geq 2$.

Theorem 3. Let $\sigma=\left(\rho, \lambda_{2}, \overline{\lambda_{2}}, \ldots, \lambda_{n}\right)$ be realizable by a nonnegative matrix $A$, where $\rho$ is the Perron root and $\lambda_{2}$ and $\overline{\lambda_{2}}$ are non-real complex conjugates. Then, given any $\epsilon>0$, the list $\left(\rho+(2+\epsilon) t, \lambda_{2}+\right.$ $\left.t, \overline{\lambda_{2}}+t, \lambda_{4}, \ldots, \lambda_{n}\right)$ is realizable for all sufficiently small $t>0$.

2010 Mathematics Subject Classification. 15A18,15A29.

Key words and phrases. Nonnegative matrices, spectrum, eigenvalues.

Received on 21-10-2012.

Support from SFIRFP is gratefully acknowledged. 
Theorem 4. If $\sigma=\left(\rho, \lambda_{2}, \ldots, \lambda_{j}, \overline{\lambda_{j}}, \ldots, \lambda_{n}\right)$, where $\rho$ is the Perron root, is realizable by a nonnegative circulant matrix, then for all $t>0$ the list $\left(\rho+c t, \lambda_{2}, \ldots, \lambda_{j}+t, \overline{\lambda_{j}}+t, \ldots, \lambda_{n}\right)$ is also realizable by a nonnegative circulant matrix for

$$
c \geq \begin{cases}2, & \text { if } n \text { is even } \\ 2 \cos \left(\frac{\pi}{n}\right), & \text { if } n \text { is odd }\end{cases}
$$

Theorem 5. Let

$$
\begin{aligned}
& f_{1}(x)=x^{n}-a_{1} x^{n-1}-\cdots-a_{n-1} x-a_{n} \\
& f_{2}(x)=x^{n}-b_{1} x^{n-1}-\cdots-b_{n-1} x-b_{n} \\
& f_{3}(x)=u_{0}+u_{1} x+\cdots+u_{n-1} x^{n-1}
\end{aligned}
$$

and

$$
A=\left(\begin{array}{cc}
C\left(f_{1}\right) & N \\
R\left(f_{3}\right) & C\left(f_{2}\right)
\end{array}\right)
$$

where $C\left(f_{i}\right)$ is the companion matrix of $f_{i}(x)$ for $i=1,2, N$ is the matrix of all zeros except for a 1 in position $(n, 1)$ and $R$ is the $n \times n$ matrix with last row $\left(u_{0}, u_{1}, \cdots, u_{n-1}\right)$ and all other rows zero. Then (a) A has characteristic polynomial $f(x):=f_{1}(x) f_{2}(x)-f_{3}(x)$, (b) if $A$ is nonnegative and $f(x)=x^{k} F(x)$ where $F(x)$ is a polynomial of degree $2 n-k$, where $0 \leq k \leq n$, then $F(x)$ is the characteristic polynomial of a $(2 n-k) \times(2 n-k)$ nonnegative matrix.

Theorem 6. Let $f(x)=x^{n}+p_{1} x^{n-1}+p_{2} x^{n-2}+\cdots+p_{n-1} x+p_{n}$ be the characteristic polynomial of a nonnegative $n \times n$ matrix $A$. Then $g(x)=\frac{f^{\prime}(x)}{n}$ is the characteristic polynomial of a nonnegative $(n-1) \times(n-1)$ matrix for $n \leq 4$ and for $n \in\{5,6\}$ when $\operatorname{tr} A=0$.

\section{Theorem 7. SNIEP $\neq$ DNIEP}

\section{REFERENCES}

[1] A.G. Cronin, T.J. LAffey, An inequality for the spectra of nonnegative matrices, Linear Algebra Appl. 436(9), (2012), 3225-3238.

[2] S. Guo, W. Guo, Perturbing non-real eigenvalues of non-negative real matrices, Linear Algebra Appl. 426, (2007), 199-203.

[3] T.J. LAffey, Perturbing non-real eigenvalues of non-negative real matrices, Electron. J. Linear Algebra 12, (2005), 73-76.

[4] V. Monov, A family of symmetric polynomials of the eigenvalues of a matrix, Linear Algebra Appl. 429, (2008), 2199-2208

School of Mathematical Sciences, University College Dublin E-mail address: anthony.cronin@ucd.ie 